\title{
Dynamic absorption coefficients of CAR and non-CAR resists at $\mathrm{EUV}$
}

\author{
Roberto Fallica* ${ }^{\mathrm{a} *}$, Jason K. Stowers ${ }^{\mathrm{b}}$, Andrew Grenville ${ }^{\mathrm{b}}$, Andreas Frommhold ${ }^{\mathrm{c}}$, \\ Alex P. G. Robinson ${ }^{\mathrm{c}}$, Yasin Ekinci ${ }^{\mathrm{a}}$ \\ ${ }^{a}$ Paul Scherrer Institute, 5232 Villigen PSI, Switzerland \\ ${ }^{\mathrm{b}}$ Inpria Corporation, $2001 \mathrm{NW}$ Monroe Ave. STE 203, 97330 Corvallis OR, USA \\ ${ }^{\mathrm{c}}$ School of Chemical Engineering, University of Birmingham, Edgbaston, Birmingham, UK
}

\begin{abstract}
The dynamic absorption coefficients of several CAR and non-CAR EUV photoresists are measured experimentally using a specifically developed setup in transmission mode at the XIL beamline of the Swiss Light Source. The absorption coefficient $\alpha$ and the Dill parameters ABC were measured with unprecedented accuracy. In general the $\alpha$ of resists match very closely with the theoretical value calculated from elemental densities and absorption coefficients, whereas exceptions are observed. In addition, through the direct measurements of the absorption coefficients and dose-to-clear values, we introduce a new figure of merit called Chemical Sensitivity to account for all the post-absorption chemical reaction ongoing in the resist, which is also predicts a quantitative clearing volume, and respectively clearing radius, due to the photon absorption in the resist. These parameters may help in deeper insight into the underlying mechanisms of EUV concept of clearing volume and clearing radius are then defined and quantitatively calculated.
\end{abstract}

Keywords: absorption coefficient, alpha, Dill parameters, Chemical Sensitivity, CS, Clearing volume, clearing radius

\section{INTRODUCTION}

In photolithographic processing, the optimum absorption is determined by the tradeoff between efficiently harvesting the incoming light (high absorption) and the need to maintain a constant intensity throughout the film thickness (low absorption). Photoresists for DUV and earlier technologies were designed to coat at 200-800 nm thickness. These resists were mostly based on DNQ and novolak resins compounds, which yield an absorption coefficient $(\alpha)$ ranging from 0.5 to $1 \mu \mathrm{m}^{-1}{ }^{1,2}$ At these values of $\alpha$, the intensity of light reaching at the bottom of the resist layer is still about $80 \%$ of the intensity at the top, which guarantees both an adequate depth clearance and a limited sidewall slope. The latter is especially important in high aspect ratio lift-off processes, where the resist sidewalls should ideally be vertical.

On the other hand, EUV photoresists are designed to coat at $<45 \mathrm{~nm}$ thickness and, despite the absorption coefficient is in the range of few $\mu \mathrm{m}^{-1}$, the total optical absorbance can be as low as $10 \%$. The low absorbance has several detrimental consequences on the lithographic process. First, EUV photons are costly and difficult to produce due to the complexity of sources and should be efficiently used. Moreover, the photon density in $13.5 \mathrm{~nm}$ wavelength photolithography is about 14 times lower than it is in $193 \mathrm{~nm}-\mathrm{ArF}$ excimer laser lithography (the dose being the same). As only about 6 photons are incident per $\mathrm{nm}^{2}$ and only 0.03 are absorbed per $\mathrm{nm}^{3}$ in a typical EUV photoresist exposed at $10 \mathrm{~mJ} / \mathrm{cm}^{2}$ dose, the photon shot noise becomes more relevant and contributes substantially to line edge roughness.

Resist manufacturers are undertaking several approaches to counterbalance the low absorbance of EUV resists. One approach is to increase the amount of secondary electrons generated per each absorbed photon (i.e. the quantum yield), which depends on the chemical composition of the resist. Another approach, specific of chemically amplified resists (CAR), is to increase the amount of photoacids generated per photon, i.e. the quantum efficiency. These solutions aim at improving the efficiency of each absorbed photon.

Another approach tackles the low absorption by increasing the absorption of the resist by incorporating highly absorbing elements. In general, the linear absorption coefficient of a compound is given by sum of the atomic absorption cross section $\sigma_{a}$ of each element, weighted for its relative amount $x_{i}$ and density $\rho_{i}$ :

*roberto.fallica@psi.ch; phone+4156310 4578; www.psi.ch

Extreme Ultraviolet (EUV) Lithography VII, edited by Eric M. Panning, Kenneth A. Goldberg. Proc. of SPIE Vol. 9776, 977612 - ( 2016 SPIE · CCC code: 0277-786X/16/\$18 · doi: 10.1117/12.2219193 


$$
\alpha=\frac{N_{A}}{M W} \sum_{i} x_{i} \rho_{i} \sigma_{a_{i}}
$$

(where $\mathrm{N}_{\mathrm{A}}$ is the Avogadro number and $\mathrm{MW}$ is the molecular weight of the compound). The most promising elements for incorporation in a photoresists are the transition metals and semimetals with large atomic numbers whose large $d$ orbitals result in the largest interaction with EUV photons as it can be seen in Figure 1 where atomic absorption cross sections for all elements of atomic number from 1 to 86 are shown.

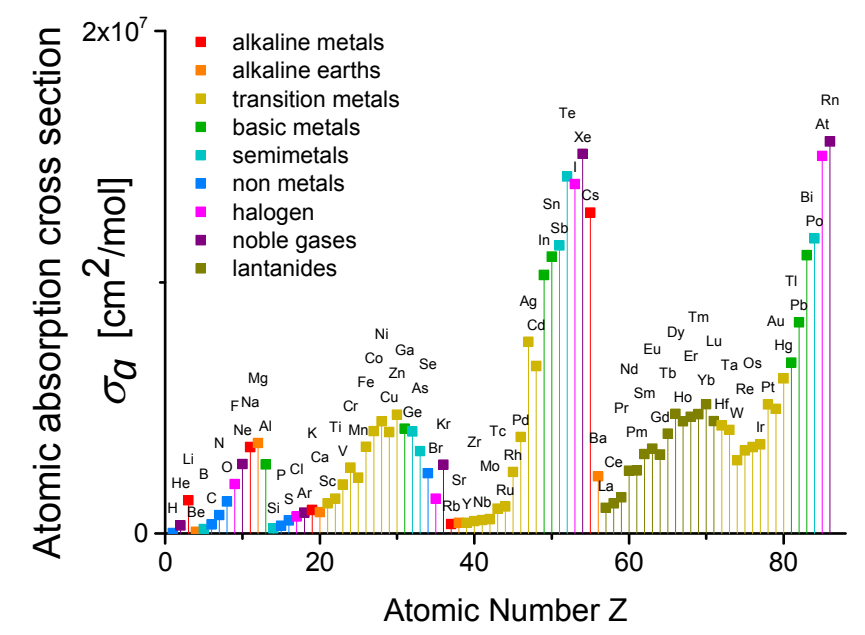

Figure 1. Atomic absorption cross-sections $\sigma_{a}$ at EUV of elements with atomic number from $\mathrm{Z}=1$ to 86 . Calculated from experimental data. ${ }^{3}$

In recent years, a great technological effort has being put towards the development of resists featuring enhanced absorption. For example, fluorinated polymers have been investigated as the backbone polymers of EUV resists ${ }^{4-6}$ owing to the large photoabsorption cross-section of fluorine atoms. Some fluorine-based resists have been reported to increase the absorption from 5.2 to $7.0 \mu^{-1}$, although the change in backbone polymers significantly affects acid generation, acid diffusion, catalytic chain reactions, and dissolution. Other studies claimed fluorinated versions of PMMA which could in principle provide up to $18 \mu \mathrm{m}^{-1}$ absorbance (up from $3.88 \mu \mathrm{m}^{-1}$ of a conventional FEVS-P1101 resist) ${ }^{7}$. The addition of metals is a viable way to synthesize negative-tone photoresists based on organometallic carboxylate compounds of the type $\left[\mathrm{RnM}\left(\mathrm{O} 2 \mathrm{CR}\right.\right.$ ')2], with $\mathrm{M}=\mathrm{Sn}, \mathrm{Te}, \mathrm{Bi}, \mathrm{Sb}^{8}$ Another method involves the synthesis of photoresists based on nanoparticle/molecular metals. The incorporation of $\mathrm{ZrO}_{\mathrm{x}}$ and $\mathrm{TeO}_{\mathrm{x}}$ nanoparticles in a CAR has been demonstrated to reduce its transmittance by up to $\approx 8 \%$ depending on the relative amount of oxide, compared to the original formulation. ${ }^{9}$

In this work we measured experimentally the static and the dynamic absorption coefficients of photoresists at EUV (13.5 $\mathrm{nm})$. Besides the static linear absorption coefficient $\alpha$, the dynamic absorption coefficients are technologically important for the accurate chemical and physical modeling of the lithographic process. These coefficients are known as the Dill parameters, or the $\mathrm{ABC}$ parameters. We chose resists belonging to a broad spectrum of platforms and we discuss these results to determine the chemical sensitivity. The sources of experimental uncertainty are accounted for, thus providing quantitative data with unprecedented accuracy.

\section{EXPERIMENTAL METHODS}

\subsection{Transmittance measurement at XIL beamline}

The XIL beamline at Swiss Light Source (SLS) employs synchrotron generated EUV light at $13.5 \mathrm{~nm}$ with average flux in excess of $\approx 30 \mathrm{~mW} / \mathrm{cm}^{2}$. This tool is mainly used for EUV interference lithography ${ }^{10}$ and lensless EUV mask imaging. For this study we made minor additions to the setup enabling to measure the intensity of the light passing through a sample in transmission mode. The experimental setup is schematically shown in Figure 2 . The beam intensity and shape was set by using a pinhole of $30 \mu \mathrm{m}$ diameter. A square open-frame mask $\left(0.5 \times 0.5 \mathrm{~mm}^{2}\right)$ was located in front of the sample to crop out the beam tail. The combination of pinhole and openframe resulted in a highly homogenous beam intensity within the exposed area. Because the membrane itself also absorbs EUV light, we calibrated the net flux $I_{0}$ 
through a blank silicon nitride membrane. The transmittance $T_{X}$ of the thin film is then given by the ratio between measured photocurrent $I$ and the reference photocurrent $I_{0}$.

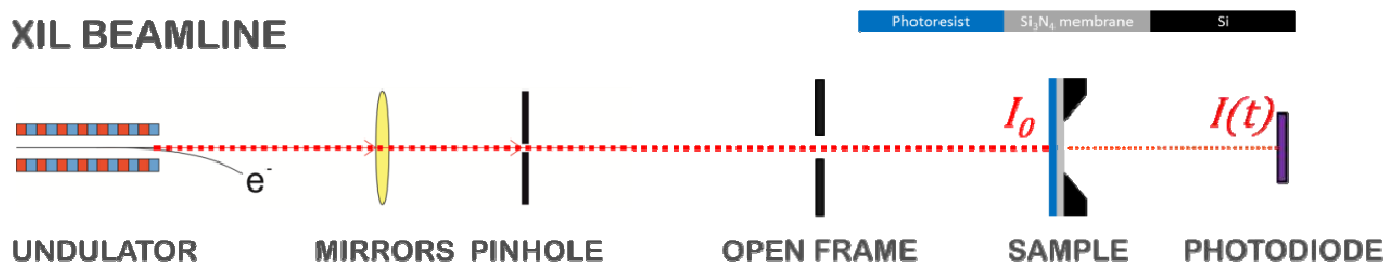

Figure 2. Schematic of experimental setup for absorption measurements.

\subsection{Linear absorption coefficient and Dill Parameters}

As the Beer-Lambert law describes, the transmittance of light through a homogeneous medium is a function of the thickness $d$ and of the linear absorption coefficient:

$$
T_{X}=e^{-\alpha d}
$$

From the previous equation, the linear absorption coefficient can be calculated as:

$$
\alpha=-\frac{1}{d} \ln \frac{I\left(t_{0}\right)}{I_{0}}\left[\mu m^{-1}\right]
$$

In our experimental setup, the current measured from the photodiode was recorded as a function of time, from the beginning $\left(t=t_{0}\right)$ and throughout the end of the exposure $\left(t=t_{\text {exp }}\right)$. Here, $t_{\exp }$ is the time needed to fully expose the resist, calculated as the ratio between flux (which is constant during the exposure) and the dose-to-clear, which is also measured with the same tool a priori. We thus calculate the bleaching of the photoresist using the definitions of Dill parameters ${ }^{11}$ for the bleachable absorption coefficient $A$ :

$$
A=\frac{1}{d} \ln \frac{I\left(t_{\text {exp }}\right)}{I\left(t_{0}\right)}\left[\mu m^{-1}\right]
$$

the unbleachable absorption coefficient $B$ :

$$
B=-\frac{1}{d} \ln \frac{I\left(t_{\text {exp }}\right)}{I_{0}}=\alpha-A\left[\mu m^{-1}\right]
$$

and the exposure rate constant $C$ :

$$
C=\left.\frac{A+B}{A \Phi\left[I(0)-I(0)^{2} / I_{0}\right]} \frac{d I}{d t}\right|_{t=0}\left[\frac{c m^{2}}{m J}\right]
$$

\subsection{Sample preparation and description}

Silicon nitride membranes $\left(100 \mathrm{~nm}\right.$ nominal thickness, $\left.3 \times 3 \mathrm{~mm}^{2}\right)$ suspended on a silicon frame $(280 \mu \mathrm{m}$ thick, $9 \times 9$ $\mathrm{mm}^{2}$ ), were fabricated by selective KOH etch of silicon wafers. Thin photoresist films were subsequently spun coated on the membranes and processed according to manufacturer specifications (pretreatment, post application bake, underlayer, etc.).

For the sensitivity (dosage curve) measurements, the same resists were also spun coated on bulk silicon wafers, $500 \mu \mathrm{m}$ thick, and processed according to manufacturer specifications.

The samples description is provided in the following Table 1. We chose the well-known PMMA and Hydrogen silsesquioxane (HSQ), as reference systems. Four chemically amplified EUV-specific photoresists, from undisclosed manufacturer and labeled here 1,2, and 3 were analyzed. A modified version of the latter resist, featuring increased sensitizer concentration was also tested (EUV 3+S). Two materials of metal oxide based photocondensed resists, nonchemically amplified, containing tin from Inpria Corp. (YABA and a YF) were measured. Finally we measured fullerene polymer based resist provided from Irresistible Materials, some of which containing metal. 
Table 1. Samples description.

\begin{tabular}{|c|c|c|c|c|}
\hline Resist & Manufacturer & Type & C.A. & Metal containing \\
\hline PMMA & Sigma Aldrich & organic & non $C A$ & $\mathrm{~N}$ \\
\hline HSQ & Dow & inorganic & non $C A$ & $\mathrm{~N}(\mathrm{HSiOx})$ \\
\hline EUV 1 & undisclosed & organic & CA & $\mathrm{N}$ \\
\hline EUV 2 & undisclosed & organic & CA & $\mathrm{N}$ \\
\hline EUV 3 & undisclosed & organic & CA & $\mathrm{N}$ \\
\hline EUV 3+S & undisclosed & organic & CA & $\mathrm{N}$ \\
\hline YABA & Inpria & metal oxide based & non CA & $Y(S n)$ \\
\hline YF & Inpria & metal oxide based & non $C A$ & $Y(S n)$ \\
\hline xMT-213 & IM & organic & CA & $\mathrm{N}$ \\
\hline PBPM & IM & organic & non $\mathrm{CA}$ & $\mathrm{Y}$ \\
\hline ML356 & IM & organic & non CA & $Y$ \\
\hline HB1-213 & IM & organic & CA & $Y$ \\
\hline
\end{tabular}

\subsection{Spectroscopic ellipsometry}

The accurate measurement of the thickness of resist coated on a suspended membrane is challenging and it is a major source of uncertainty in the estimation of $\alpha$. Resist thickness were measured using a Woollam M-2000 spectroscopic ellipsometer with spectral range $250-1000 \mathrm{~nm}$ and equipped with focusing probe to reduce the spot size to about $30 \mu \mathrm{m}$ diameter. Samples were mounted on an automatic motorized stage and the entire membrane was mapped with a 175 micron step grid. The spot for the ellipsometer was a $92 \mu \mathrm{m}$ x $42 \mu \mathrm{m}$ oval. The ellipsometric data was modeled as two layers, for the $\mathrm{SiN}$ membrane a B-spline dispersion model seeded with $\mathrm{Si}_{3} \mathrm{~N}_{4}$ material properties and for the resist a Cauchy model. Initial material fits were generated from the silicon frame surrounding the suspended membrane. Neither additional layers nor interfacial were needed to obtain a good fitting of the thickness. This approach greatly reduces the uncertainty in the $\alpha$, using a combination of optical inspection and spectroscopic ellipsometry to estimate $d$ in the proximity of the exposed area.

\subsection{Estimation of uncertainty}

The uncertainty in the measurement of $\alpha$ arises from three sources. The first is the uncertainty in the measurement of the resist thickness $\left(\sigma_{d}\right)$, due to the non uniformity of the coating. The second is the uncertainty in the thickness of the silicon nitride membrane, due to the $\mathrm{KOH}$ etch variability, which affects the value of the reference photocurrent $\left(\sigma_{I 0}\right)$. The third is the uncertainty in the measured photocurrent from the photodiode $\left(\sigma_{I}\right)$. The standard deviation of $\alpha$ is then given by the well-known formula for error propagation and replacing the due quantities:

$$
\sigma_{\alpha}=\sqrt{\left(\frac{\partial \alpha}{\partial d}\right)^{2} \sigma_{d}^{2}+\left(\frac{\partial \alpha}{\partial I_{0}}\right)^{2} \sigma_{I_{0}}^{2}+\left(\frac{\partial \alpha}{\partial I(t)}\right)^{2} \sigma_{I(t)}^{2}} \cong \sqrt{\left(\frac{\partial \alpha}{\partial d}\right)^{2} \sigma_{d}^{2}+\left(\frac{\partial \alpha}{\partial I_{0}}\right)^{2} \sigma_{I_{0}}^{2}}=\sqrt{\alpha^{2} \frac{\sigma_{d}^{2}}{d^{2}}+\frac{1}{d^{2}} \frac{\sigma_{I_{0}}^{2}}{I_{0}^{2}}}
$$

From experimental data, the fluctuations in measured photocurrent were negligible in comparison to the other terms of Eq. 7, and therefore were not taken into account. 


\section{RESULTS AND DISCUSSION}

\subsection{Linear absorption coefficient $\alpha$}

The measured transmittance of all samples is plotted in Fig. 3 as a function of resist thickness $d$. Resists that were coated at different thickness showed a linear relation between the logarithm of transmittance and $d$, which indicates a homogeneous optical absorption through the medium. The linear absorption coefficient $\alpha$ can thus be determined as the slope of the linear fit to the data. The linear fit is weighted by the X-axis error bar which indicates the uncertainty in the thickness measurement $\sigma_{d}$ and by the Y-axis error bar which indicates the uncertainty in the transmittance $\sigma_{I o}$. (For those resists spin coated at only one thickness, we consider the line through the origin).

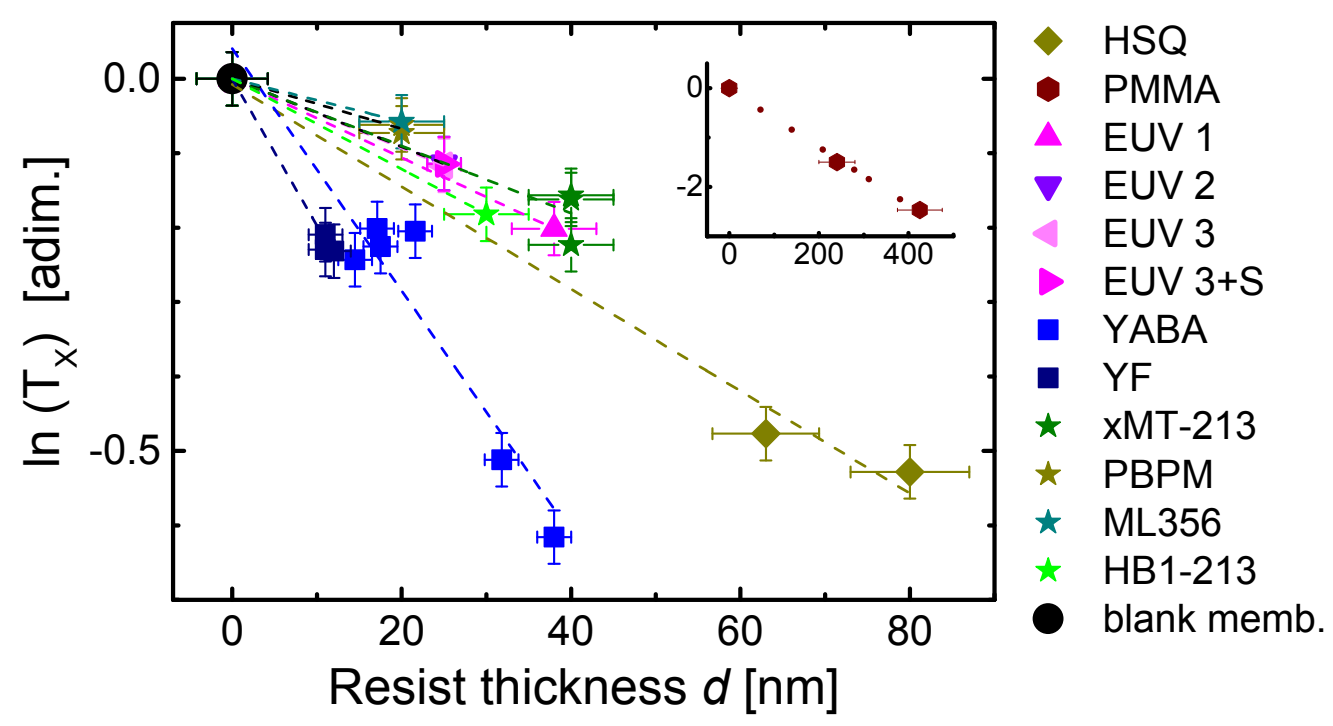

Figure 3. Logarithm of measured transmittance as a function of resist film thickness. For each measurement point, the Xaxis error bar indicates the uncertainty in the thickness measurement $\sigma_{d}$ and the Y-axis error bar indicates the uncertainty in the transmittance $\sigma_{I o}$. The dashed lines are the linear fit to the data. The slope of the fit is the linear absorption coefficient $\alpha$. Inset: measured data for PMMA.

The measured $\alpha$ is summarized in the following Figure 4; the error bar is calculated from the error-weighted linear fit of the $\mathrm{T}_{\mathrm{X}}$ vs. thickness plot.

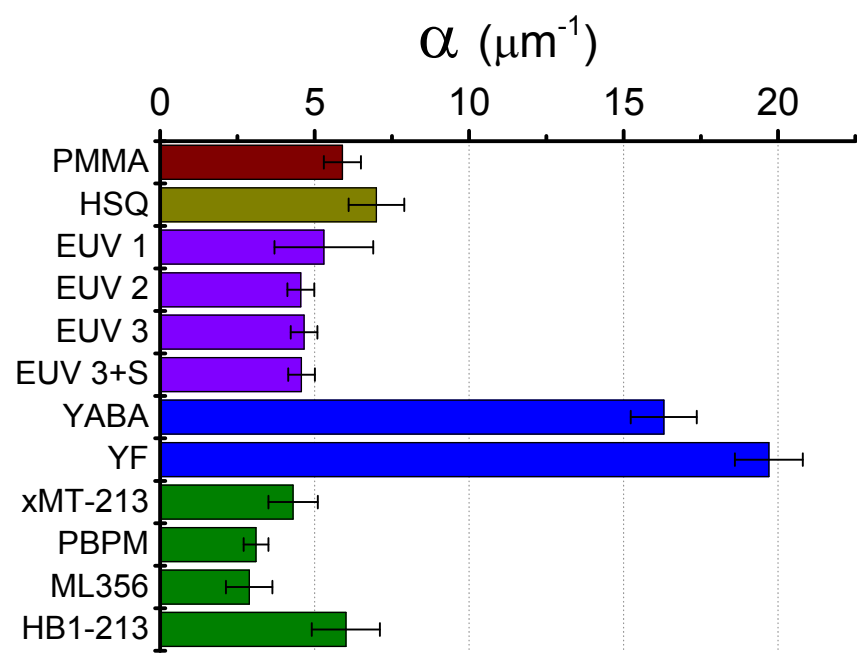

Figure 4. Measured linear absorption coefficient $\alpha$. All polymer-based resist have approximately the same value, while the tin oxide-based resists (YABA and YF) have a remarkably higher $\alpha$. 
The photoresists based on the tin oxide (YABA and YF) showed the highest absorption values, while all the other resist platforms are 3 to 4 times lower. Previous theoretical studied predicted that resists based on polymeric backbones only achieve $\alpha$ values in the 3 to $5 \mu \mathrm{m}^{-1}$ range, ${ }^{12}$ as a consequence of the chemical composition, where only $\mathrm{C}$ and $\mathrm{O}$ atoms contribute to the absorption. Our result for PMMA is in good agreement with previous experimental studies, which reported that its EUV absorption is between $\approx 5 \mu \mathrm{m}^{-1},{ }^{13}$ and $4.8 \mu \mathrm{m}^{-1}{ }^{12}$ Note that the CAR resist with added sensitizer (EUV $3+\mathrm{S}$ ) had the same absorption coefficient as its baseline formulation (EUV 3). Other resists with added metal elements also showed higher absorption, $6 \mu \mathrm{m}^{-1}$ (HB1-213), than that of conventional CAR resists. We conclude that the absorption increases most remarkably only in metal-added non-polymer based resists. Our values are in agreement with those reported on some typical polymer backbones for EUV, between 2.5 and $5.2 \mu \mathrm{m}^{-1}$ measured using the grazing incidence angle method. ${ }^{14}$

To validate our results we calculated the theoretical absorption coefficient of these resists using the online database of Xray interactions, ${ }^{15}$ based on tabulated data of X-ray absorption published previously. ${ }^{3}$ The chemical composition and density used were for PMMA: $\mathrm{C}_{5} \mathrm{O}_{2} \mathrm{H}_{8}$, molecular weight 950k, dilution $1 \%$, density $1.2 \mathrm{~g} / \mathrm{cm}^{3}$; for $\mathrm{HSQ}_{2} \mathrm{Si}_{8} \mathrm{O}_{12} \mathrm{H}_{8}$, density $1.4 \mathrm{~g} / \mathrm{cm}^{3}$; for proprietary materials, values provided by the manufacturer. The comparison between our experimental data and the calculated data shows a close match between the two (Fig. 5).

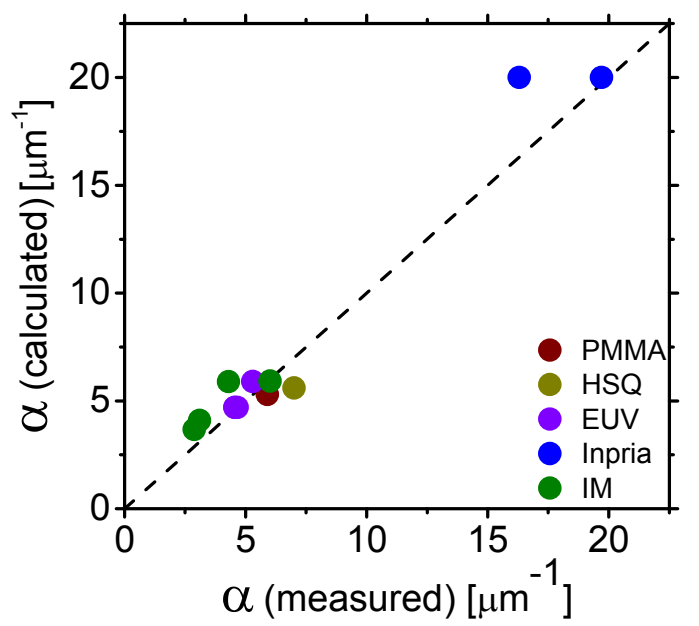

Figure 5. Comparison between experimental and theoretical absorption coefficient for all resists in this work. The dash line indicates the exact match.

\subsection{Dill Parameters}

The measured Dill parameters (also known as the ABC parameters) are of interest for the lithographic modeling. We summarized these results in the following Figure 6.

For all resists investigated in this work, and in general for EUV resists, the bleachable coefficient $A$ was found to be positive and much smaller than $\alpha$. The Dill parameter $A$ can also indicate a change in the resist thickness during exposure. As a result of being $A$ « $\alpha$, the unbleachable coefficient $B \approx \alpha$. This behavior is notably different to that of Iline and G-line resists, which have $A \gg B .^{1}$ 


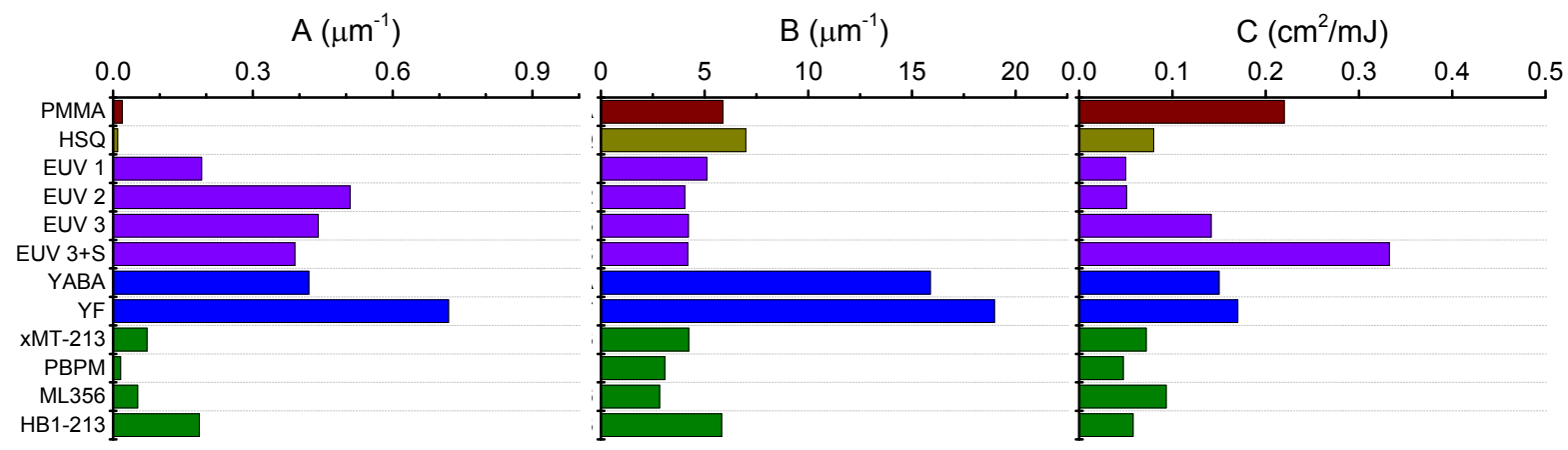

Figure 6. Summary of the measured Dill parameters: bleachable coefficient A , unbleachable coefficient B, and exposure rate constant $\mathrm{C}$ of resists investigated in this work.

The Dill parameter $C$ is proportional to the rate of bleaching at $t=0$, and it is an empirical indicator of the rate of reaction of the resist at the beginning of exposure cause by, among other things, deprotection reactions and mass loss due to outgassing. It was found that the CAR resist with added sensitizer (EUV 3+S) yielded a significantly higher reaction rate than its baseline counterpart (EUV 3). In a previous study, other researchers found $C$ parameter values of 0.04 $\mathrm{cm}^{2} / \mathrm{mJ}$ for a anion-bound polymer bound CAR using film thickness loss curves method. ${ }^{16}$ Another study by Fourier transform infrared spectroscopy gave a reaction rate of $0.0409 \pm 0.0023 \mathrm{~cm}^{2} / \mathrm{mJ}$ for another undisclosed CAR, ${ }^{17}$ in good agreement with our result. Other methods reported $C$ in the range of $0.037-0.055 \mathrm{~cm}^{2} / \mathrm{mJ}^{18-20}$ In comparison to previous technological platforms, the Dill $C$ parameter is higher in EUV than it was in UV lithography $\left(0.022 \approx 0.008 \mathrm{~cm}^{2} / \mathrm{mJ}\right)^{1}$ and in DUV lithography $0.005 \mathrm{~cm}^{2} / \mathrm{mJ}^{2}$

\subsection{Chemical Sensitivity}

In the light of the new understanding of the absorption coefficient $\alpha$, we introduce here a new figure of merit. First we observe that the lithographic sensitivity of a resist is proportional to the reciprocal of its dose to clear (DtC) or dose to gel:

$$
\text { LithoSens }=\frac{1}{D t C}
$$

Furthermore, we observe that the lithographic sensitivity is proportional to the product of the amount of absorbed photons (via the absorption coefficient) and the combination of quantum efficiency, quantum yield, chemical amplification and so on (i.e. all chemical reaction which take place after a photon is absorbed):

$$
\text { LithoSens }=\alpha \times \sum(Q E, Q Y, C A, \text { etc. })
$$

By rearranging Eq.8 and Eq. 9, we define 'Chemical Sensitivity' (CS) the quantity:

$$
C S=\frac{1}{\alpha \times D t C}
$$

CS accounts for the contribution that all secondary chemical reactions make to the overall lithographic sensitivity. Because $\alpha$ is expressed in $\mu \mathrm{m}^{-1}$ and DtC is expressed in $\mathrm{mJ} / \mathrm{cm}^{2}, \mathrm{CS}$ is in units of volume per energy $\left(\mathrm{m}^{3} / \mathrm{J}\right)$ or, more intuitively, in cubic nanometers per unit energy of an EUV photon $\left(\mathrm{nm}^{3} / \mathrm{E}_{\mathrm{ph}}\right)$. In this interpretation, the chemical sensitivity indicates the volume of photoresist that is cleared by a single absorbed EUV photon. CS is larger when each absorbed photon triggers a more significant amount of secondary chemical reactions which clear (or condense) a larger resist volume. As it rules out the absorption and the thickness of a material, the CS can be compared across different resist platforms.

The CS was calculated using Eq. 10 from measured $\alpha$ and DtC (from contrast curves) for all these resists and the results are shown in Fig. 7. All CARs have CS of about several tens of cubic nanometers per photon energy: most of their lithographic sensitivity is caused by a high chemical sensitivity. On the opposite, all non-CAR resists have CS below 10 $\mathrm{nm}^{3} / \mathrm{E}_{\mathrm{ph}}$, regardless of any other feature. In agreement with our reasoning, we notice that the CS increases when sensitizer is added to a CAR resist (EUV $3+\mathrm{S}$ ) in comparison to the same baseline formulation (EUV 3). 


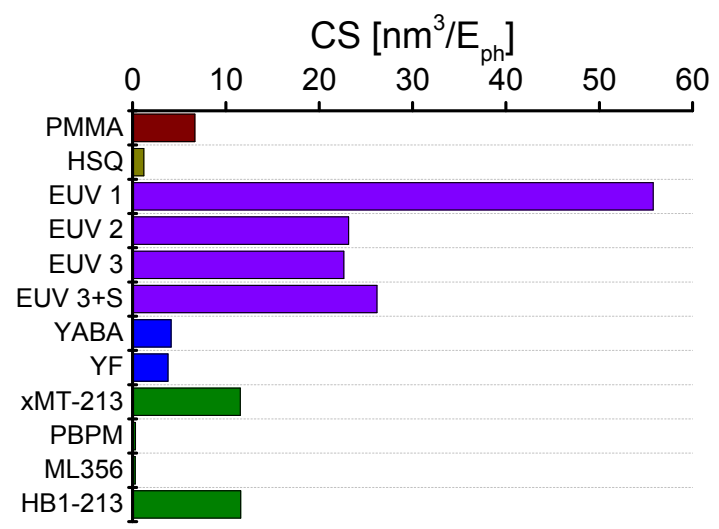

Figure 7. Chemical sensitivity of resists, calculated from measured $\alpha$ and dose-to-clear values as described in Eq. 10.

Higher chemical sensitivity is on one hand desirable to increase the lithographic sensitivity, but on the other hand it sets an ultimate limit to the maximum resolution at which a resist can be patterned. To clarify this, let us consider the sphere of resist having the volume equal to $\mathrm{CS}$; its radius (clearing radius) indicates the width of the clearing triggered by an absorbed photon and its secondary reactions. The calculated clearing radiuses for all resists are shown in the following Figure 8 .

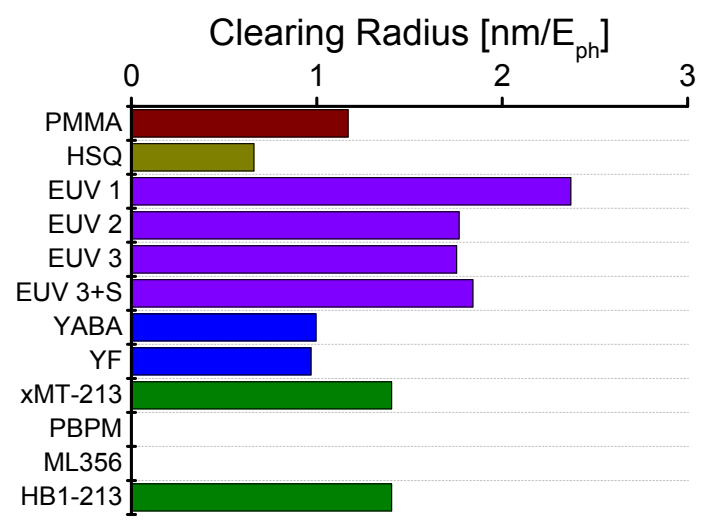

Figure 8. Clearing radius of the sphere having the volume of $\mathrm{CS}$, for each resist.

For the reasons discussed, the clearing radius is obviously larger in CARs than it is in non-CARs. In the former, the spatial range is in fact increased due to the acid diffusion. In the latter, instead, the radius is only caused by the secondary electron blur. In this regard, the clearing radius is an indication of the total resist blur. This interpretation agrees with previous theoretical and experimental studies which reported a SEB range of $\approx 2.5 \mathrm{~nm}^{21,22}$ and an acid diffusion blur $\geq 5$ $\mathrm{nm}^{16,21,22}$.

The measured $\alpha$ and the newly introduced CS make it possible to draw some conclusions. Figure 9 shows the resist map where each of the samples is now located according to its $\alpha$ and CS. The area of each sample is proportional to the total lithographic sensitivity. The map shows how different resist platforms are being designed to improve the absorption or enhance the chemical sensitivity. The only resist in this work which combines these two approaches is HB1-213. Although higher absorption and higher chemical sensitivity bring the advantage of a higher lithographic sensitivity, there are also drawbacks. On one hand, increasing the former leads to a non-uniform absorption throughout the resist thickness; while, increasing the latter reduces the resolution. In other words, these resists are still limited by the RLS tradeoff. 


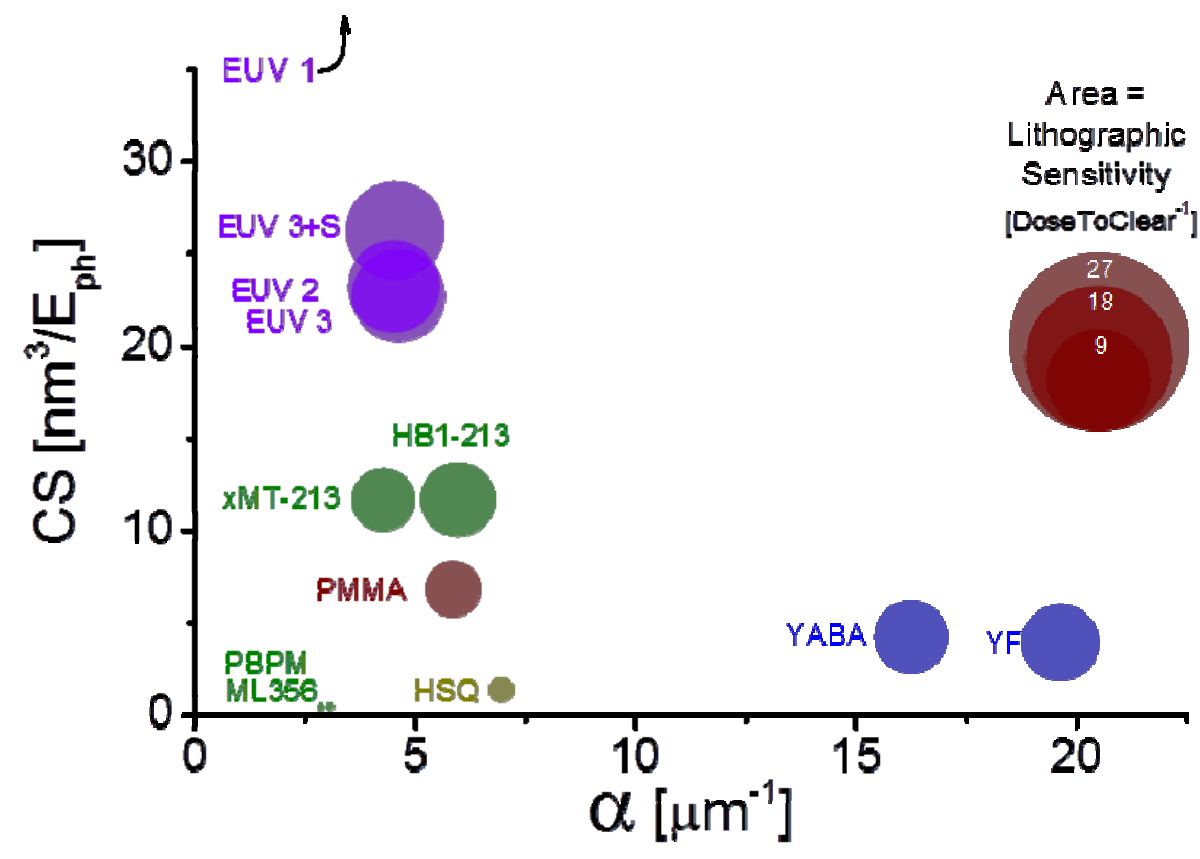

Figure 9. Location of resist according to absorption $\alpha$ and chemical sensitivity CS. The area of the bubbles is proportional to the total lithographic sensitivity.

\section{CONCLUSIONS}

We developed a methodology for the measurement of the absorption coefficient $\alpha$ and Dill parameters of photoresists at EUV. Our results are consistent with those reported previously for PMMA, HSQ, and with theoretical estimation. Metal oxide-based resists absorb up to $\mathrm{x} 4$ more photons than polymer based resists. In all resists studied in this work, the bleachable $A$ coefficient is positive but much smaller than the non-bleachable coefficient $B$.

Using our insight of the absorption and total sensitivity, we introduced a new figure of merit which makes it possible to quantify the efficiency of post-absorption reactions to the total sensitivity of a material. This chemical sensitivity is significantly higher in chemically amplified resists than in non-chemically amplified resists. Finally, our methodology provides insight on the two approaches towards higher lithographic sensitivity in EUV photoresists.

\section{ACKNOWLEDGEMENTS}

The Authors are grateful to Irresistible Materials Ltd., for sample preparation and fruitful discussion, and to Markus Kropf (PSI) for technical support. Part of this work has been carried out at SLS. One of the Authors (R. F.) wishes to acknowledge Inpria Corp. for financial support.

\section{REFERENCES}

[1] MicroChemicals, "Optical Properties of Photoresists," Application Note (2013).

[2] Sohn, Y.-S., Oh, H.-K., and An, I., "Parameter extraction for $193 \mathrm{~nm}$ chemically amplified resist from refractive index change," Journal of Vacuum Science \& Technology B 19(6), 2077-2081 (2001). 
[3] Henke, B. L., Gullikson, E. M., and Davis, J. C., "X-ray interactions: photoabsorption, scattering, transmission, and reflection at E=50-30000 eV, Z=1-92," Atomic Data and Nuclear Data Tables 54(2), 181-342 (1993).

[4] Christianson, M. D., Meyer, M. M., Ongayi, O., Valeri, D., and Wagner, M., "High Absorbing Resists Based on Trifluoromethacrylate-Vinyl Ether Copolymers for EUV Lithography," SPIE Advanced Lithography 868216 (2013).

[5] Kwark, Y.-J., Bravo-Vasquez, J. P., Chandhok, M., Cao, H., Deng, H., Gullikson, E., and Ober, C. K., "Absorbance measurement of polymers at extreme ultraviolet wavelength: Correlation between experimental and theoretical calculations," Journal of Vacuum Science \& Technology B 24, 1822 (2006).

[6] Sekiguchi, A., Matsumoto, Y., Harada, T., Watanabe, T., and Kinoshita, H., "Study of Dill's B parameter measurement of EUV resist," 94222L-94222L-94214 (2015).

[7] Gronheid, R., Fonseca, C., Leeson, M. J., Adams, J. R., Strahan, J. R., Willson, C. G., and Smith, B. W., "EUV Resist Requirements: Absorbance and Acid Yield," SPIE Advanced Lithography (2009).

[8] Passarelli, J., Murphy, M., Del Re, R., Sortland, M., Dousharm, L., Vockenhuber, M., Ekinci, Y., Neisser, M., Freedman, D. A., and Brainard, R. L., "High-sensitivity molecular organometallic resist for EUV (MORE)," SPIE Advanced Lithography 94250T-94251 (2015).

[9] Sekiguchi, A., Matsumoto, Y., Yamanouchi, T., Watanabe, T., Harada, T., and Kudo, H., "A Study of EUV Resist Sensitivity by Using Metal Materials," International Symposium on EUV Lithography.

[10] Mojarad, N., Gobrecht, J., and Ekinci, Y., "Beyond EUV lithography: a comparative study of efficient photoresists' performance," Sci. Rep. 5((2015).

[11] Dill, F. H., Hornberger, W. P., Hauge, P. S., and Shaw, J. M., "Characterization of positive photoresist," IEEE Transactions on Electron Devices ED-22(445-452 (1975).

[12] Matsuzawa, N. N., Oizumi, H., Mori, S., Irie, S., Yano, E., Okazaki, S., and Ishitani, A., "Theoretical estimation of absorption coefficient of various polymers at 13 nm," Microelectronic Engineering 53(671-674 (2000).

[13] Kubiak, G. D., and al., e., "Characterization of chemically amplified resists for soft x-ray projection lithography," Journal of Vacuum Science \& Technology B 10(2593 (1992).

[14] Kwark, Y.-J., Bravo-Vasquez, J. P., Chandhok, M., Cao, H., Deng, H., Gullikson, E., and Ober, C. K., "Absorbance measurement of polymers at extreme ultraviolet wavelength: Correlation between experimental and theoretical calculations," Journal of Vacuum Science \& Technology B 24(4), 1822-1826 (2006).

[15] The Center for X-ray Optics, L. B. N. L. s., http://henke.lbl.gov/optical_constants/ by Eric Gullikson, (1995).

[16] Gronheid, R., Vaglio Pret, A., Rathsack, B., Hooge, J. S., Scheer, S., Nafus, K., Shite, H., and Kitano, J., "Resolution-linewidth roughness-sensitivity performance tradeoffs for an extreme ultraviolet polymer bound photo-acid generator resist," J. Micro/Nanolith. MEMS MOEMS 10(1), 013017 (2011).

[17] Lee, C.-T., Yueh, W., Roberts, J., and Henderson, C. L., "A Simple Method for Measurement of Photoacid Generator Photoreaction Kinetics in Formulated, Chemically Amplified Photoresist Films," Electrochemical and Solid-State Letters 10(9), H273-H277 (2007).

[18] Szmanda, C. R., Brainard, R. L., Mackevich, J. F., Awaji, A., Tanaka, T., Yamada, Y., Bohland, J., Tedesco, S., Dal'Zotto, B., Bruenger, W., Torkler, M., Fallmann, W., Loeschner, H., Kaesmaier, R., Nealey, P. M., and Pawloski, A. R., "Measuring acid generation efficiency in chemically amplified resists with all three beams," Journal of Vacuum Science \& Technology B 17(6), 3356-3361 (1999).

[19] Pawloski, A. R., Szmanda, C. R., and Nealey, P. F., "Evaluation of the standard addition method to determine rate constants for acid generation in chemically amplified photoresist at $157 \mathrm{~nm}, " 1056-1065$ (2001).

[20] Cameron, J. F., Chan, N., Moore, K., and Pohlers, G., "Comparison of acid-generating efficiencies in 248 and 193nm photoresists," 106-118 (2001).

[21] Kozawa, T., and Tagawa, S., "Thermalization Distance of Electrons Generated in Poly(4-hydroxystyrene) Film Containing Acid Generator upon Exposure to Extreme Ultraviolet Radiation," Japanese Journal of Applied Physics 50(030209 (2011).

[22] Thackeray, J. W., Wagner, M., Jin Kang, S., and Biafore, J. J., "Understanding the Role of Acid vs. Electron Blur in EUV Resist Materials," Journal of Photopolymer Science and Technology 23(5), 631-637 (2010). 